\title{
Primary total knee replacement using dished polyethylene with resected posterior cruciate ligament
}

\author{
Mohan M. Kumar, Satvik N. Pai*, Pravin K. Vanchi, Raghav Ravi, Syam Nath
}

Department of Orthopaedic Surgery, Sri Ramachandra Medical College and Research Institute, Chennai, India

Received: 15 May 2021

Revised: 10 June 2021

Accepted: 11 June 2021

\author{
*Correspondence: \\ Dr. Satvik N. Pai, \\ E-mail: satvik.pai@gmail.com
}

Copyright: () the author(s), publisher and licensee Medip Academy. This is an open-access article distributed under the terms of the Creative Commons Attribution Non-Commercial License, which permits unrestricted non-commercial use, distribution, and reproduction in any medium, provided the original work is properly cited.

\begin{abstract}
Background: The choice between preserving, sacrificing or substituting the posterior cruciate ligament (PCL) is always a controversial topic in total knee replacement (TKR). Dished polyethylene insert with PCL resection enables correction of the commonly present fixed flexion and varus deformities. Additionally, the risk of premature wear of polyethylene is less because of the confirming articular geometry between the femoral and tibial component.

Methods: This is a retrospective study in which we studied 120 knees in 95 consecutive patients undergoing primary TKR by the senior author at our institute. We used TKR system with dished metal backed polyethylene tibial component. PCL resection was performed in all cases. Pre-operative and post-operative functional assessment were done using knee society clinical scores and Western Ontario and McMaster universities osteoarthritis index (WOMAC). All radiographs were assessed using the knee society Roentgenographic scoring system (KSRES). Statistical analysis was performed using paired student $t$ tests. Survivorship was determined using Kaplan-Meier survivorship curves.

Results: Mean follow-up was 8 years. Range of motion increased from 75 degrees to 110 degrees. The knee society pain score increased from 30 to 94 . The knee society function score increased from 35 to 75 . WOMAC score increased in terms of pain, stiffness and physical function.

Conclusions: We conclude that deep dish bearing is a viable option in presence of deficient PCL and provides adequate stability and functional outcome. We need a larger sample size, multicentre trial and longer follow-up to see for complication rate, revision rate and survival.
\end{abstract}

Keywords: Total knee replacement, Dished poly insert, Midterm results

\section{INTRODUCTION}

The choice between preserving, sacrificing or substituting the PCL is always a controversial topic in total knee replacement (TKR). The benefits of preserving the PCL are retained femoral rollback, superior knee kinematics and improved stair-climbing ability. ${ }^{1}$ The patients are also likely to have better range of movement of the knee joint. Conforming articular surfaces minimize premature wear of the polyethylene. The drawback of preserving the PCL are higher peak contact forces, abnormal screw home motion, eccentric loading and possible premature polyethylene lining wear. ${ }^{2}$ There is also the need for additional resection of femoral bone at the intercondylar notch, which is a problem in most of the small built Asian women population.

The possible solution is to use dished polyethylene insert with PCL resection (Figure 1 and 2). It enables correction of the commonly present fixed flexion and varus deformities. ${ }^{3}$ Additionally, the risk of premature wear of polyethylene is less because of the confirming articular geometry between the femoral and tibial component. ${ }^{4}$ The aim of our study was to determine the functional and radiological outcomes of PCL sacrificing TKR using dished polyethylene inserts. 


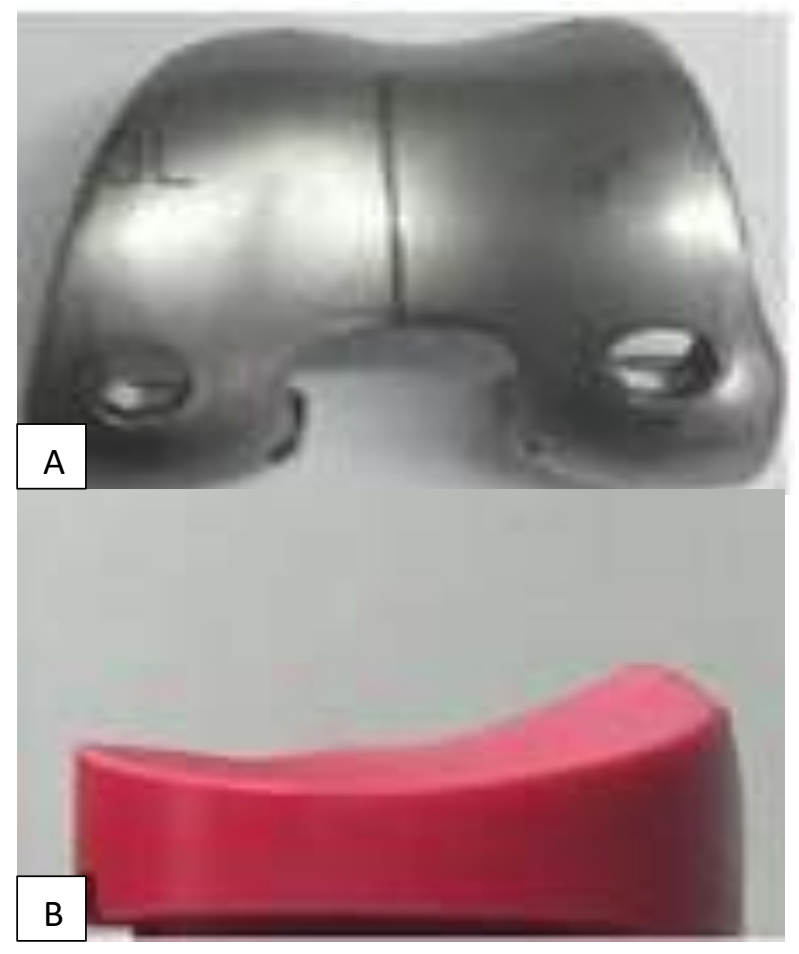

Figure 1: Cruciate retaining femoral component with ultra-congruent deep dished polyethylene (trial).
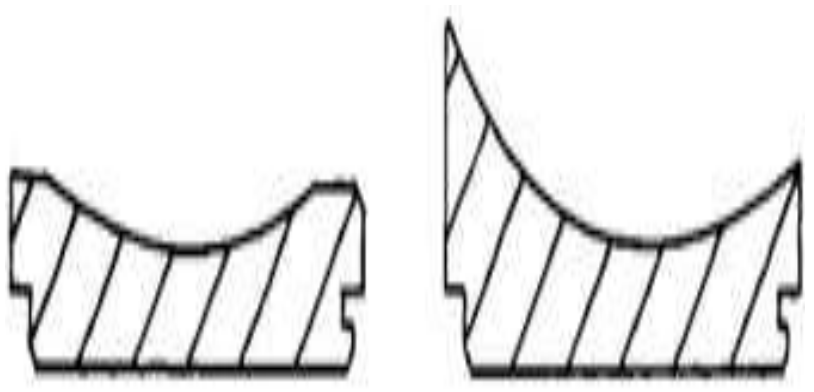

Figure 2: Anterior build-up in deep dished polyethylene in comparison to flat polyethylene insert.

\section{METHODS}

This is a retrospective study approved by the institutional ethics committee at our institution. This study involved 120 knees in 95 consecutive patients undergoing primary TKR by the senior author at Sri Ramachandra medical college and research institute during the period of January 2008- December 2015. All patients having follow up more than 5 years were included in the study. Any patients lost to follow up earlier than 5 years post operatively were excluded from the study. We used TKR system with dished metal backed polyethylene tibial component (Smith-Nephew, Genesis II system). The Genesis II system includes a cobalt chromium femoral component, titanium tibial component with tibial insert consisting of a dished ultrahigh molecular weight polyethylene liner. Patelloplasty was done all cases.

The surgical approach consisted of a midline incision followed by a medial parapatellar arthrotomy. Patients received perioperative third generation cephalosporin. PCL resection was performed in all cases. All prostheses were implanted using hand-mixed cement fixation of the femoral, tibial components. All varus-valgus deformities were corrected using appropriate soft tissue releases. Postoperative rehabilitation protocol for all patients included immediate weight bearing as tolerated on post-operative day 1. Low-molecular-weight heparin was used for deep vein thrombosis prophylaxis till the pts were actively mobilised. Patients were discharged on the $5^{\text {th }}$ postoperative day.

Preoperative and post-operative functional assessment was done using knee society clinical scores, Western Ontario and McMaster universities osteoarthritis index (WOMAC). Radiographic assessments were also done. Weight-bearing anteroposterior, lateral, and patellofemoral radiographs were obtained preoperatively and at each postoperative follow-up visit. All radiographs were evaluated by a single experienced surgeon and were assessed using the knee society roentgenographic scoring system (KSRES). The prosthesis-bone interface was evaluated for the presence and progression of radiolucent lines. Progression of a radiolucent line was defined as an increase in length and/or width of $2 \mathrm{~mm}$ or greater on sequential radiographs. Component alignment and position were compared with previous radiographs to determine whether subsidence or loosening had occurred. Joint spaces, as evaluated on anteroposterior weightbearing radiographs, were evaluated for asymmetry or changes in thickness suggestive of polyethylene insert wear.

Statistical analysis was performed using paired Student $\mathrm{t}$ tests (with significance set at $\mathrm{p}<0.05$ ). Survivorship was determined using Kaplan-Meier survivorship curves, with clinical or radiographic failure (defined as a revision of the tibial, femoral components or any subsequent procedure that altered knee components) taken as the end point. SPSS software was used for statistical analysis.

\section{RESULTS}

The study comprised 66 females and 29 males. Mean age at the time of operation was 65.2 years (range, 45-80 years); mean weight was $80 \mathrm{~kg}$ (range, 75-95 kg). Mean time from index surgery to most recent follow-up was 8 years (range, 5.5 to 12 years). 25 cases were performed as bilateral procedures. Pre-operative diagnoses were osteoarthritis (94\%) and rheumatoid arthritis (6\%).

Of the original 97 patients (122 knees), 2 patients died of causes unrelated to the surgery. At a mean follow-up of 8.2 years (range, 6-12 years), ROM had increased from a preoperative mean of $75^{\circ}$ (range, $25^{\circ}-120^{\circ}$ ) to $110^{\circ}$ (range, 
$\left.75^{\circ}-130^{\circ}\right)$. Knee society pain and function scores increased from means of 30 (range, 4-69) and 35 (range, 0-80) to 94 (range, 51-100) and 75 (range, 30-100), respectively. Results were good or excellent in $90 \%$ of knees (100 excellent, 8 good); 5 patients reported fair results and 3 patients had a poor outcome due to comorbid diseases. WOMAC analysis, graded on a scale of 0 to 4 , with 4 representing severe impairment, improved from mean normalized scores of 3 for pain (range, 1-4), 2.8 for stiffness (range, 0-4), and 3.1 for physical function (range, 0.18-4) preoperatively to 0.6 (range, 0-3), 0.4 (range, 0 3.5 ), and 0.7 (range, 0-3.35), respectively at most recent follow-up.

Radiographically, there were no changes in prosthetic alignment or position. There was no gross evidence of thinning or asymmetry consistent with wear or failure of the tibial or femoral components (Figure 3, 4 and 5). On AP x-rays, 6 knees $(5 \%)$ exhibited radiolucent lines in knee society zone 1 of the tibial component-bone interface; On lateral rays 2 knees exhibited radiolucent lines in zone 1 of the tibial component-bone interface. All observed radiolucency's were less than $2 \mathrm{~mm}$ and were nonprogressive in subsequent films.

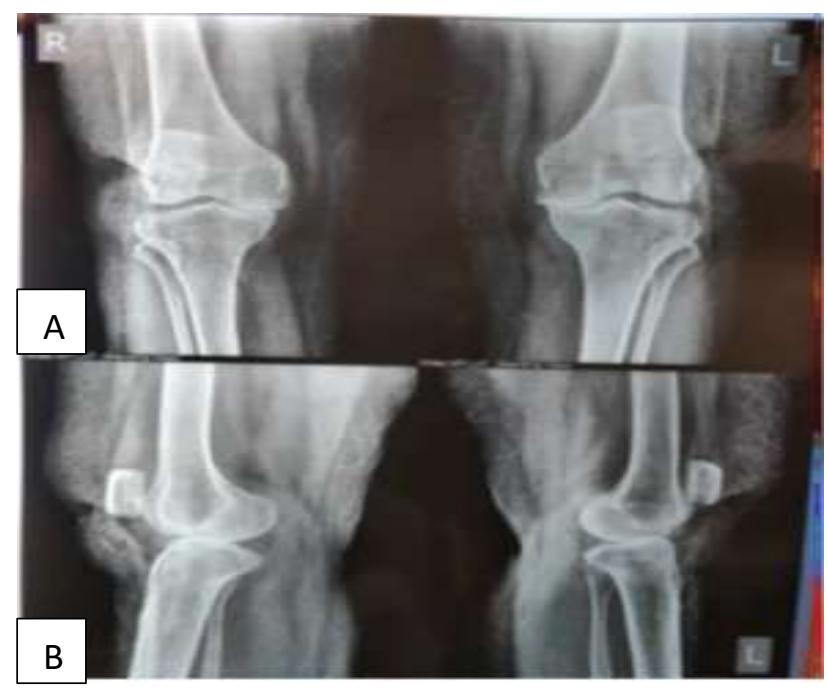

Figure 3: Pre-operative radiograph of bilateral OA knee patient with genu varum deformity.

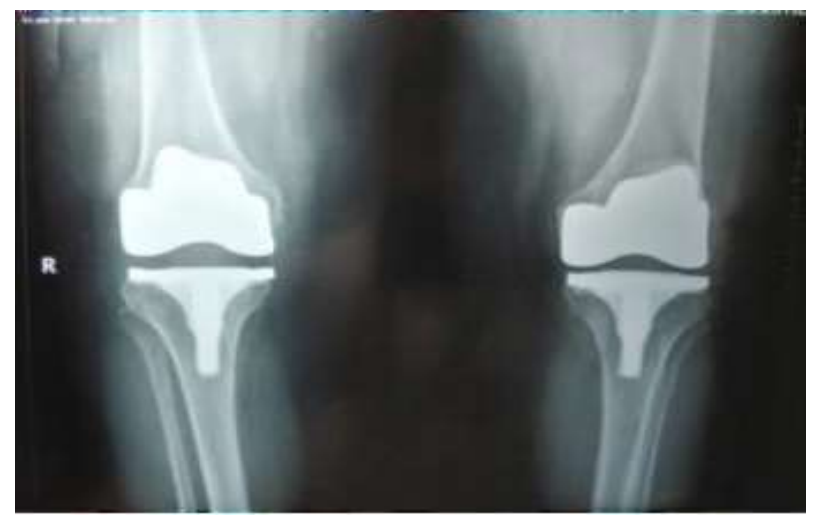

Figure 4: 7 years post-operative AP radiograph.

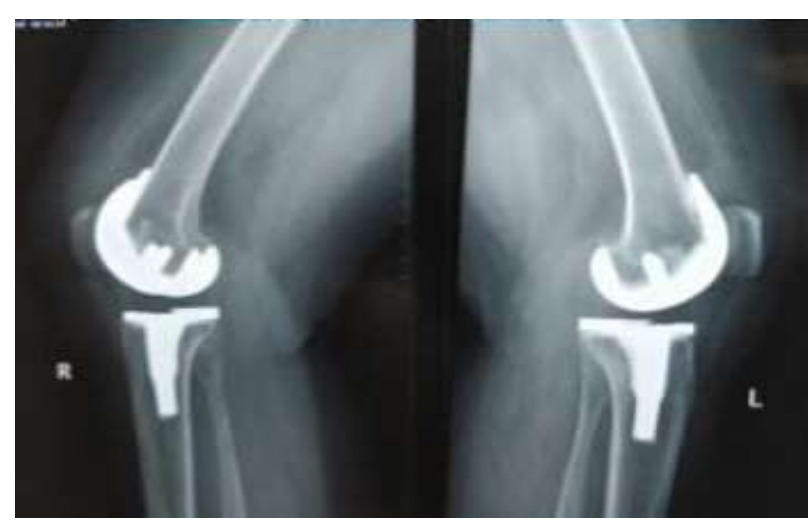

Figure 5: 7 years post-operative lateral radiograph.

2 patients underwent revision. One patient, who suffered a fall 1 year after surgery, sustained a periprosthetic fracture of the lateral femoral condyle and underwent a successful lateral femoral column reconstruction with LCP. This patient is functioning well for 8 years. A second revision followed a fall in which the patient sustained a tibial plateau fracture 14 months after the primary TKA. This patient had failed 4 months of conservative treatment after the accident; subsequently, serial radiographs revealed medial subsidence and loosening of the tibial component. This patient underwent successful tibial component revision with a long-stem implant and wedge augmentation; 8 years later, the patient had no complaints of pain knee society (KSS) score 95, but ambulated with a cane due to severe osteoarthritis of the contralateral knee.

\section{DISCUSSION}

This study aimed to explore whether deep dished polyethylene bearing prosthesis provides adequate stability in the presence of completely released PCL. The benefits of the deep dished design include ease of conversion from cruciate retaining (CR) design to PCL substituting design and bone preservation. It has also been proposed to have improved wear characteristics resulting from the increased surface contact area and potentially decreased surface contact forces. There have been drawbacks reports as well. Deep dished bearings could result in an inability to restore normal knee kinematics and the potential to create a kinematic conflict.

We performed a search of literature on the outcomes of TKRs using dished polyethylene tibial inserts. Laskin et al performed a comparison between posterior stabilized tibial polyethylene insert versus dished polyethylene tibial insert and found no statistically significant differences in ROM, ability to ascend and descend stairs, or knee scores. ${ }^{5}$ Scott and Thornhill in their study comparing flatter and less conforming polyethylene insert with sagittal curved and more conforming polyethylene insert in TKA's, showed that dished or curved insert provide more contact area and lesser stress than its flatter counterparts. ${ }^{6}$ In their study they concluded that the dished inserts do not adversely affect ROM or tibial radiolucency compared 
with flat tibial inserts. Font-Rodriguez et al studied the long-term results of 215 total condylar prostheses with an all-polyethylene dished tibia. ${ }^{7}$ They reported an average annual failure rate of $0.46 \%$ and a 21 -year success rate of $90.77 \%$. These studies suggest that the theoretical disadvantages of using a dished component when retaining the PCL, namely kinematic conflict or instability, do not apply clinically in the situation where the PCL is rendered incompetent by either resection or recession. Sathappan et al did a retrospective analysis of 114 knees with ultracongruent deep dished polyethylene insert TKR's with a recessed or resected PCL. ${ }^{8}$ There were no statistically significant differences $(\mathrm{p}<0.05)$ between PCL recession and resection cases with regard to ROM, Knee Society scores, or WOMAC scores. They concluded that excellent functional outcome could be achieved with dished polyethylene designs, which is sufficient for anteroposterior knee stability when proper balancing of flexion and extension gap is done and mechanical axis of the knee is restored. Bartel et al supports the usage of dished polyethylene insert in their study on normal cadaveric knees. ${ }^{9}$ During axial loading, the dished polyethylene inserts result in a wedging effect that produces increased stability between the tibial and femoral surfaces. They also inferred that during stress and strain analysis on several knee designs, there is reduction in interface stress and polyethylene wear in systems with conforming articulating surfaces and concluded that they were superior to other articulations they studied. Comparison of our results with those of other studies has depicted in Table 1.

Table 1: Comparison of mean pre op and follow up range of movement (ROM), KSS for pain, and

Function score (FS) in our study and other studies.

\begin{tabular}{|lllll|}
\hline Variables & $\begin{array}{l}\text { Our } \\
\text { study }\end{array}$ & $\begin{array}{l}\text { Sathappan } \\
\text { et al }^{\mathbf{8}}\end{array}$ & $\begin{array}{l}\text { Parsley } \\
\text { et al }^{\mathbf{1 0}}\end{array}$ & $\begin{array}{l}\text { Serna } \\
\text { et al }^{11}\end{array}$ \\
\hline $\begin{array}{l}\text { Age } \\
\text { (years) }\end{array}$ & 59.91 & 68.4 & 67.5 & 70.1 \\
\hline $\begin{array}{l}\text { Pre op } \\
\text { ROM }\end{array}$ & 74.15 & 92 & 105.3 & 90 \\
\hline $\begin{array}{l}\text { Follow } \\
\text { up ROM }\end{array}$ & 108.3 & 111 & 116.7 & 100.7 \\
\hline $\begin{array}{l}\text { Pre op } \\
\text { KSS }\end{array}$ & 40.85 & 35.2 & 45.7 & 36.4 \\
\hline $\begin{array}{l}\text { Pre op } \\
\text { FS }\end{array}$ & 43.72 & 39.7 & 44.1 & 47.2 \\
\hline $\begin{array}{l}\text { Follow } \\
\text { up KKS }\end{array}$ & 86.91 & 91.3 & 86.3 & 85.2 \\
\hline $\begin{array}{l}\text { Follow } \\
\text { up FS }\end{array}$ & 87.23 & 74.7 & 64.5 & 85.6 \\
\hline
\end{tabular}

Deep dished inserts aimed to avoid the cam-mechanism wear and femoral inter-condylar bone sacrifice related to posterior stabilised inserts. As per the available literature and our experience in this study, the functional and radiological outcomes of deep-dish inserts are comparable to other options at mid-term follow-up, with the additional benefit of bone conservation. The preservation of good quality bone in intercondylar notch prevents supracondylar fracture of distal femur. It is particularly important in small size femurs encountered in most Asian women. It also decreases operative time, by avoiding the time required for intercondylar bone resection. During revision of posterior stabilised knees, it is very often difficult to remove the bone cement near the box. This problem can however be avoided by using deep dished poly with cruciate retaining femur design.

\section{CONCLUSION}

We conclude that deep dish bearing is a viable option in presence of deficient PCL and provides adequate stability and functional outcome. We need a larger sample size, multicentre trial and longer follow-up to see for complication rate, revision rate and survival.

\section{Funding: No funding sources \\ Conflict of interest: None declared}

Ethical approval: The study was approved by the institutional ethics committee

\section{REFERENCES}

1. Dorr LD, Ochsner JL, Gronley J. Functional comparison of posterior cruciate-retained versus cruciate-sacrificed total knee Arthroplasty, Clin Orthop. 1988;36.

2. Hirsch HS, Lotke PA, Morrison LD. The posterior cruciate ligament in total knee surgery. Save, sacrifice, or substitute? Clin Orthop. 1994;64.

3. Dennis DA, Komistek RD, Stiehl JB. Range of motion after total knee arthroplasty: the effect of implant design and weight-bearing conditions. J Arthroplasty. 1998; $13: 748$.

4. Soudry M, Walker PS, Reilly DT. Effects of total knee replacement design on femoral-tibial contact conditions. J Arthroplasty. 1986;1:35.

5. Laskin RS, Maruyama Y, Villaneuva M. Deep dish congruent tibial component use in total knee arthroplasty: a randomized prospective study. Clin Orthop. 2000;36.

6. Scott RD, Thornhill TS. Posterior cruciate supplementing total knee replacement using conforming inserts and cruciate recession. Effect on range of motion and radiolucent lines. Clin Orthop. $1994 ; 146$.

7. Font-Rodriquez DE, Scuderi GR, Insall JN. Survivorship of cemented total knee arthroplasty. Clin Orthop. 1997;79.

8. Sathappan SS, Bradley W, William LJ. Midterm results of primary total knee arthroplasty using a dished polyethylene insert with recessed or resected posterior cruciate ligament. J Arthroplasty. 2006;21:1012.

9. Bartel DL, Rawlinson JJ, Burstein AH. Stresses in polyethylene components of contemporary total knee replacements. Clin Orthop. 1995;76.

10. Parsley BS, Conditt MA, Bertolusso R, Noble PC. Posterior cruciate ligament substitution is not 
essential for excellent postoperative outcomes in total knee arthroplasty. J Arthroplasty. 2006;21(6-2):12731.

11. Serna-Berna R, Lizaur-Utrilla A, Vizcaya-Moreno MF, Miralles Muñoz FA, Gonzalez-Navarro B, Lopez-Prats FA. Cruciate-Retaining vs PosteriorStabilized Primary Total Arthroplasty. Clinical
Outcome Comparison with a Minimum Follow-Up of 10 Years. J Arthroplasty. 2018;33(8):2491-5.

Cite this article as: Kumar MM, Pai SN, Vanchi PK, Ravi R, Nath S. Primary total knee replacement using dished polyethylene with resected posterior cruciate ligament. Int J Res Orthop 2021;7:781-5. 